\title{
Sampling Directed Graphs with Random Walks
}

\author{
Bruno Ribeiro $^{1}$, Pinghui Wang ${ }^{2}$, Fabricio Murai ${ }^{1}$, and Don Towsley ${ }^{1}$ \\ ${ }^{1}$ Computer Science Department \\ University of Massachusetts \\ Amherst, MA, 01003 \\ \{ribeiro, fabricio, towsley\}@cs.umass.edu \\ ${ }^{2}$ State Key Lab for Manufacturing Systems \\ Xi'an Jiaotong University \\ Xi' an P.R.China \\ phwang@sei.xjtu.edu.cn
}

\begin{abstract}
Despite recent efforts to characterize complex networks such as citation graphs or online social networks (OSNs), little attention has been given to developing tools that can be used to characterize directed graphs in the wild, where no pre-processed data is available. The presence of hidden incoming edges but observable outgoing edges poses a challenge to characterize large directed graphs through crawling, as existing sampling methods cannot cope with hidden incoming links. The driving principle behind our random walk (RW) sampling method is to construct, in real-time, an undirected graph from the directed graph such that the random walk on the directed graph is consistent with one on the undirected graph. We then use the RW on the undirected graph to estimate the outdegree distribution. Our algorithm accurately estimates outdegree distributions of a variety of real world graphs. We also study the hardness of indegree distribution estimation when indegrees are latent (i.e., incoming links are only observed as outgoing edges). We observe that, in the same scenarios, indegree distribution estimates are highly innacurate unless the directed graph is highly symmetrical.
\end{abstract}

\section{INTRODUCTION}

Despite recent efforts to characterize complex networks such as citation graphs or online social networks (OSNs), little attention focused on developing tools for characterizing directed graphs in the wild, where no pre-processed data is available. A network is said to be directed when the relationships between its agents (users or profiles) may not be reciprocated. For instance, a Wikipedia [18] entry about Columbia Records cites Thomas Edison but Thomas Edison's entry makes no reference to Columbia Records.

The presence of hidden incoming edges but observable outgoing edges makes characterizing large directed graphs through crawling a challenge. An edge $b \rightarrow a$ is a hidden incoming edge of node $a$ if $b \rightarrow a$ can only be observed from node $b$. For instance, in our earlier Wikipedia example about Columbia Records and Thomas Edison we cannot observe the edge "Columbia Records" $\rightarrow$ "Thomas Edison" from Thomas Edison's wiki entry (but this edge is observable if we access Columbia Records's wiki entry).

Unless we can crawl the entire graph, hidden incoming edges may induce unknown biases in the estimation process. Moreover, there may not even be a directed path from a given node to all other nodes. Graphs with hidden outgoing edges but observable incoming edges exhibit essentially the same problem. For instance, in some countries individuals, politicians, and NGOs may be required to publicly disclose their contributors but contributors are not required to publicly list their contributions.
In this work we propose a random walk sampling algorithm that obtains asymptotically unbiased estimates of the outdegree distribution of a directed graph. Our experiments indicate that crawling even a small fraction of the graph allows us to accurately estimate the outdegree distribution of a variety of real world graphs. Our random walk algorithm resorts to two main principles to achieve unbiased samples:

- In real-time we construct an undirected graph using the directed nodes that are sampled by the random walker on the directed graph. The role of the undirected graph is to guarantee that at the end of the sampling process we can approximate the probability of sampling a node, even though incoming edges are not observed. The random walk proceeds in a way that the sample path of walking on the directed graph is consistent with the sample path of a classical random walk on the constructed undirected graph. This allows us to generate an asymptotically unbiased estimate of the outdegree distribution.

- A limited number of uniformly sampled nodes (less than 0.01 of all sampled nodes) to guarantee that different parts of the directed graph are explored.

\section{Contributions}

Our work makes two main contributions:

- Directed Unbiased Random Walk (DURW): Our random walk algorithm accurately estimates characteristics of large directed graphs through sampling.

- In-degree Distribution Estimation: We show experimentally that no unbiased estimator can accurately obtain the indegree distribution (recall indegrees are latent variables in the directed graph) of the datasets used in this work from sampled edges unless a large fraction of the graph is sampled and/or the directed graph is highly symmetric. This result is surprising as the average indegree and average outdegree are identical and the outdegree distribution can be accurately characterized.

\section{Outline}

The rest of the paper is organized as follows. Section II presents the graph model and some definitions used throughout this work. Section III presents our DURW algorithm and estimators. Section IV presents our outdegree distribution estimation simulation results on real world graphs. Section V presents an application of $D U R W$ on the Wikipedia network. Section VI shows that indegree distribution estimation is 
inaccurate unless most of the graph is sampled (even with side information). Section VII reviews the related work. Finally, Section VIII presents our conclusions and future work.

\section{Definitions and Problem Formulation}

Let $G_{d}=\left(V, E_{d}\right)$ be a directed graph, where $V$ is the set of nodes and $E_{d}$ is the set of edges. Let $o(v)$ denote the number of edges out of node $v \in V$ (outdegree) and $i(v)$ denote the number of edges into node $v \in V$ (indegree). We seek to obtain both the outdegree distribution $\phi=\left(\phi_{0}, \phi_{1}, \ldots, \phi_{R}\right)$ and the indegree distribution $\theta=\left(\theta_{0}, \theta_{1}, \ldots, \theta_{W}\right)$, where $\phi_{l}$ is the fraction of nodes with outdegree $l, \theta_{j}$ is the fraction of nodes with indegree $j, R$ is the largest outdegree, and $W$ is the largest indegree.

The degree distribution of a large undirected graph can be estimated using random walks (RW) [7], [11], [14]. But these RW methods cannot be readily applied to directed graphs with hidden incoming edges, which is the case of a number of interesting directed networks, e.g., the WWW, Wikipedia, and Flickr.

To address these problems, we build a random walk with jumps under the assumption that nodes can be sampled uniformly at random from $G_{d}$ (something not feasible for the WWW graph but possible for Wikipedia and Flickr). But why perform a random walk if we can sample nodes uniformly? There are two reasons for that: (1) Random walk is more efficient in networks where uniform node sampling is costly (e.g., Flickr). We denote the cost of sampling a random node as $c$. In networks where users have numeric IDs, the cost of uniform sampling comes from the fact that the ID space is sparsely populated [5], [6], [13] and a number of uniformly generated ID values are invalid. In these networks $c$ is the average number of IDs queried until one valid ID is obtained. For instance, in the case of MySpace and Flickr, we estimate these costs to be $c=10$ [13] and $c=77$ (refer to our technical report [15]), respectively. We consider the cost of sampling an unsampled neighboring node to be one and assume that we have a total sampling budget of $B$. (2) A random walk can better characterize highly connected nodes than uniform sampling as random walks are biased to sample highly connected nodes. This bias can be later removed, giving us smaller estimation errors for the characteristics of highly connected nodes.

\section{SAMPLing DiRECTED GRAPHS WITH DURWS}

In what follows we detail our DURW algorithm. Estimating characteristics of undirected graphs with random walks (RWs) is the subject of a number of recent works [11], [14], [16]. RW estimation methods presented in the literature require that $\forall u, v \in V$, the probability of eventually reaching $u$ given that the walker is in $v$ be non-zero. However, over a directed graph with hidden incoming edges this may not be true. For instance, consider a node $v \in V$ that has one outgoing edge but no incoming edges. If the random walker does not start at $v$ then $v$ is not visited by the walker (as the outgoing edge of $v$ is a hidden incoming edge if some other node). On the other hand, a node $u \in V$ with no outgoing edges becomes a sink to the random walker.
A natural way to deal with the unreachability of nodes is to perform random jumps within the random walk, just like the PageRank algorithm [4]. The PageRank walker at node $v$ jumps to a uniformly chosen node in the graph with probability $\alpha$; and with probability $(1-\alpha)$ the walker performs a RW step (i.e., follows an edge chosen uniformly at random from the set of outgoing edges of $v$ ). PageRank, however, does not allow us to accurately estimate graph characteristics, such as the outdegree distribution, from a sampled subset of the graph. This is because there is no simple way to obtain the stationary distribution of PageRank without knowing the complete underlying graph (we exemplify this inability in our technical report [15]). Thus, in what follows we propose DURW.

\section{A. Directed Unbiased Random Walk (DURW)}

Our Directed Unbiased Random Walk (DURW) algorithm has two parts:

- Backward edge traversals (detailed in Section III-B): In this part of the algorithm an undirected graph is constructed to allow the walker to traverse known outgoing edges backwards. Backward edge traversal happens under certain conditions, however. For instance, if at the $i$-th step the RW is at node $s_{i}$, we allow the random walker to traverse the edge $s_{i-1} \rightarrow s_{i}$ backwards. We place these restrictions on which edges can be traversed in reverse in order to avoid large transients.

- Degree-proportional jumps (detailed in Section III-C): The algorithm performs a jump from node $v$ to an uniformly chosen node, $\forall t \in V$, with probability $w /(w+$ $\operatorname{deg}(v))$, where $\operatorname{deg}(v)$ is the degree of $v$ in the undirected graph $G_{u}$. Our jumping algorithm is subtly but fundamentally different than other random jump algorithms such as PageRank.

\section{B. Backward edge traversals}

We allow the walker to traverse some outgoing edges backwards. In general, if we apply this "backward walking" principle to all outgoing edges in $G_{d}$, we could construct an undirected version of $G_{d}$. However, as incoming edges are unobservable, such universal backward traversal is not possible. The undirected version of $G_{d}$ allows us to apply the techniques described in Ribeiro and Towsley [14] to estimate the characteristics of $G_{d}$ such as the outdegree distribution. However, the degree of a node $v$ in the final undirected version of $G_{d}$ is only known after exploring all edges of $G_{d}$. Thus, the above sampling algorithm is not practical as unbiasing the sampled would require access to the complete underlying graph (as the probability is a function of $v$ 's degree [14]).

To avoid this problem our RW interactively builds an undirected graph $G_{u}$. This building process is such that once a node is visited at the $i$-th step no additional edges are added to that node in subsequent steps. Such a restriction fixes the degree of the nodes visited by the random walker, thus ensuring that nodes will not keep changing their degrees as we walk the graph. This is an important feature to reduce the random walk transient and thus reduce estimation errors. Note 
that the final undirected graph $G_{u}$ depends on the sample path taken by the random walker. Further details of the algorithm can be found in Section III-D.

The above solution addresses the problem of knowing the degree of a node as soon as the node is sampled. However, we still do not know the steady state distribution of the RW when we add random jumps. In what follows we present an algorithm that allows us to obtain a simple closed-form solution to the steady state distribution.

\section{Degree-proportional jumps}

Let $G_{u}=\left(V, E_{u}\right)$ be an undirected graph. In DURW, the probability of randomly jumping out of a node $v, \forall v \in V$, is $w /(w+\operatorname{deg}(v)), w>0$. This modification is based on the following observation: let $G^{\prime}$ be a weighted undirected graph formed by adding a virtual node $\sigma$ to $G_{u}$ such that $\sigma$ is connected to all nodes in $V$ with edges having weight $w$. All remaining edges have unitary weight. In a weighted graph a walker transverses a given edge with probability proportional to the weight of this edge. The steady state probability of visiting a node $v$ on $G^{\prime}$ is $(w+\operatorname{deg}(v)) /(\operatorname{vol}(V)+w|V|)$, where $\operatorname{vol}(V)=\sum_{\forall u \in V} \operatorname{deg}(u)$. Thus, except for the unknown constant normalization term $(\operatorname{vol}(V)+w|V|)$, the steady state distribution of $v$ is known since we know the degree of $v$ and the value of $w$ when $v$ is visited by the random walker.

By combining backward edge traversal (Section III-B) and degree-proportional jumps (Section III-C) we obtain the DURW algorithm.

\section{The DURW algorithm}

DURW is a random walk over a weighted undirected connected graph $G_{u}=\left(V, E_{u}\right)$, which is built on-the-fly. The algorithm works as follows. We build an undirected graph using the underlying directed graph $G_{d}$ and the ability to perform random jumps. Let $G^{(i)}=\left(V^{(i)}, E^{(i)}\right)$ be the constructed undirected "graph" at DURW step $i$, where $V^{(i)}$ is the node set and $E^{(i)}$ is the edge set. We call $G^{(i)}$ a "graph" because we allow $E^{(i)}$ to have edges of nodes that are not in $V^{(i)}$. Denote $G_{u} \equiv \lim _{i \rightarrow \infty} G^{(i)}$. In what follows we describe the construction of $G^{(i)}$.

Let $v \in V$ be the initial node in the random walk. Let $\mathcal{N}(v)$ denote the outgoing edges of $v$ in $G_{d}$ and let node $\sigma$ denote the virtual node representing a random jump. The random walk starts at $s_{1} \in V$. We initialize $G^{(1)}=\left(\left\{s_{1}\right\}, E^{(1)}\right)$, where $E^{(1)}=\mathcal{N}\left(s_{1}\right) \cup\{(u, \sigma): \forall u \in V\}$, where $\{(u, \sigma): \forall u \in V\}$ is the set of all undirected virtual edges to virtual node $\sigma$ (this construct of adding edges to $\sigma$ is introduced to simplify our exposition, in practice we do not need to add virtual edges to $\sigma)$. The random walker proceeds as follows.

We start with $i=1$; at step $i$ the random walker is at node $s_{i}$. Let

$$
W(u, v)= \begin{cases}w & \text { if } u=\sigma \text { or } v=\sigma \\ 1 & \text { otherwise }\end{cases}
$$

denote the weight of edge $(u, v), \forall(u, v) \in E^{(i)}, i=1,2, \ldots$. The next node, $s_{i+1}$, is selected from $E^{(i)}$ with probability $W\left(s_{i}, s_{i+1}\right) / \sum_{\forall\left(s_{i}, v\right) \in E^{(i)}} W\left(s_{i}, v\right)$. Upon selecting $s_{i+1}$ we update $G^{(i+1)}=\left(V^{(i)} \cup\left\{s_{i+1}\right\}, E^{(i+1)}\right)$, where

$$
E^{(i+1)}=E^{(i)} \cup \mathcal{N}^{\prime}\left(s_{i+1}\right),
$$

and

$\mathcal{N}^{\prime}\left(s_{i+1}\right)=\left\{\left(s_{i+1}, v\right): \forall\left(s_{i+1}, v\right) \in \mathcal{N}\left(s_{i+1}\right)\right.$ s.t. $\left.v \notin V^{(i)}\right\}$

is the set of all nodes $(u, v)$ in $\mathcal{N}\left(s_{i+1}\right)$ where node $v$ is not already in $V^{(i)}$. Note that $\mathcal{N}^{\prime}\left(s_{i+1}\right) \subseteq \mathcal{N}\left(s_{i+1}\right)$. By using $\mathcal{N}^{\prime}\left(s_{i+1}\right)$ instead of $\mathcal{N}\left(s_{i+1}\right)$ in equation (1) we guarantee that no nodes in $V^{(i)}$ change their degrees, i.e., $\forall v \in V^{(i)}$ the degree of $v$ in $G^{(i)}$ is also the degree of $v$ in $G_{u}$. Thus, we comply with the requirement presented in Section III-B that once a node $v, \forall v \in V$, is visited by the RW no edges can be added to the graph with $v$ as an endpoint.

The traversal of edges in $G^{(i)}, i=1,2, \ldots$, that connect all nodes to the virtual node $\sigma$ is easily emulated using uniform node sampling. Thus, we do not need to take the virtual node $\sigma$ into account in our calculations.

Space complexity: The space required to store $G^{(i)}$ is $O(|E|)$, where $|E|$ is the number of edges in the graph.

\section{E. Outdegree Distribution Estimator}

In this section we use the nodes visited (sampled) by our $D U R W$ algorithm to estimate the outdegree distribution. The estimator presented in this section can be easily extended to obtain the distribution of node labels, as detailed in Section III-F.

Let $s_{i}$ denote the $i$-th node visited by DURW, $i=1, \ldots, n$, $n \geq B$. Let $\phi_{j}$ be the fraction of nodes with outdegree $j$ in $G_{d}$. Let $\pi(v)$ be the steady state probability of sampling node $v$ in $G_{u}, \forall v \in V$. The outdegree distribution is estimated as

$$
\hat{\phi}_{j}=\frac{1}{n} \sum_{i=1}^{n} \frac{h_{j}\left(s_{i}\right)}{\hat{\pi}\left(s_{i}\right)}, j=0,1, \ldots
$$

where $h_{j}(v)$ is the indicator function

$$
h_{j}(v)= \begin{cases}1 & \text { if the outdegree of } v \text { in } G_{d} \text { is } j, \\ 0 & \text { otherwise }\end{cases}
$$

and $\hat{\pi}\left(s_{i}\right)$ is an estimate of $\pi\left(s_{i}\right): \hat{\pi}\left(s_{i}\right)=\left(w+\operatorname{deg}\left(s_{i}\right)\right) S$. Here $\operatorname{deg}(v)$ is the degree of $v$ in $G^{(\infty)}$ and

$$
S=\frac{1}{n} \sum_{i=1}^{n} \frac{1}{w+\operatorname{deg}\left(s_{i}\right)} .
$$

The following theorem states that $\hat{\pi}\left(s_{i}\right)$ is asymptotically unbiased.

Theorem 3.1: $\hat{\pi}\left(s_{i}\right)$ is an asymptotically unbiased estimator of $\pi\left(s_{i}\right)$.

Proof: To show that $\hat{\pi}\left(s_{i}\right)$ an asymptotically unbiased we invoke Theorem 4.1 of Ribeiro and Towsley [14], yielding $\lim _{B \rightarrow \infty} S=|V| /\left(\left|E^{(\infty)}\right|+|V| w\right)$ almost surely. Thus, $\lim _{B \rightarrow \infty} \hat{\pi}\left(s_{i}\right)=\pi\left(s_{i}\right)$ almost surely. Taking the expectation of (2) in the limit $B \rightarrow \infty$ yields $E\left[\lim _{B \rightarrow \infty} \hat{\phi}_{j}\right]=\phi_{j}$, which concludes our proof. 


\section{F. Estimating other metrics}

In a more general setting we seek to estimate the distribution obtained by the function

$$
h_{j}(v)= \begin{cases}1 & \text { node } v \text { has label } j, \\ 0 & \text { otherwise. }\end{cases}
$$

where labels can indicate any characteristics of the nodes.

In order to estimate the fraction of nodes with label $j$, we substitute the values $h_{j}\left(s_{i}\right), s_{i}=1, \ldots, n, n \geq B$ into equation (2). Here $\hat{\pi}$ is computed in the same way as before and $E\left[\lim _{B \rightarrow \infty} \hat{\phi}_{j}\right]=\phi_{j}$, still holds.

Now that we have an asymptotically unbiased estimator and it is left to test the accuracy of DURW in a variety of real world graphs.

\section{EXPERIMENTAL RESULTS}

This section compares the outdegree distribution estimates obtained by DURW against estimates obtained by the random walk algorithm of Bar-Yossef et al. [3] (presented in Section VII) and independent uniform node sampling (UNI). Our experiments are performed on a variety of real world graph datasets. The statistics of these datasets are summarized in Table I.

We now describe each dataset. Flickr, LiverJournal, and YouTube are popular photosharing, blog, and video sharing websites, respectively. In these websites a user (node) can subscribe to other user (nodes) updates forming a directed edge. Wikipedia is a free encyclopedia written collaboratively by volunteers. Each registered user has a talk page, that she and other users can edit in order to communicate and discuss updates to various articles on Wikipedia. Nodes in the WikiTalk dataset represent Wikipedia users and a directed edge from node $u$ to node $v$ represents that user $u$ edited a talk page of user $v$ at least once. The Web-Google dataset was released in 2002 by Google as a part of Google Programming Contest, where nodes represent web pages and directed edges represent hyperlinks between them [1]. Further details of the Flickr, LiverJournal, and YouTube datasets can be found in Mislove et al. [10].

Table I: Overview of directed graph datasets used in our simulations.

\begin{tabular}{c|rrccc}
\hline Graph & \# nodes & \# edges & E[out-deg] & symmetry & Type \\
\hline Flickr [10] & $1,715,255$ & $22,613,981$ & 18.1 & 0.38 & OSN \\
YouTube [10] & $1,138,499$ & $4,945,382$. & 5.3 & 0.21 & OSN \\
LiveJournal [10] & $5,204,176$ & $77,402,652$ & 18.7 & 0.27 & OSN \\
Wiki-Talk [2] & $2,394,385$ & $5,021,410$ & 3.9 & 0.86 & usr talk \\
Web-Google [1] & 875,713 & $5,105,039$ & 9.87 & 0.69 & Web \\
\hline
\end{tabular}

\section{Error Metric}

Before proceeding to our results we introduce the error metric used to compare the different sampling methods. Our primary metric of interest is the outdegree distribution because it is present in all of our datasets. Let

$$
\operatorname{NMSE}\left(\hat{\phi}_{j}\right)=\frac{\sqrt{E\left[\left(\hat{\phi}_{j}-\phi_{j}\right)^{2}\right]}}{\phi_{j}}, j=1,2, \ldots,
$$

be a measure of the relative error of the estimate $\hat{\phi}_{j}$ with respect to its true value $\phi_{j}$. Because we use the relative error. We consider values as large as $\operatorname{NMSE}\left(\hat{\phi}_{j}\right)=1$ to be acceptable regardless of the value of $\phi_{j}$. Let $c$ denote the cost of UNI which is also the cost of a random jump (the average number of IDs queried until one valid ID is obtained). For instance, Flickr has a random node sampling cost of $c=77$ (as observed in the experiments presented in [15]). Let $B$ denote the sampling budget (when $c=1, B$ is the number of distinct sampled nodes). Because we create an undirected graph on the side, multiple visits to the same node counts as just one unit of the sampling budget. Also, before proceeding to our results, it is important to note that, in DURW, the probability of performing a random jump increases with $w$ (in the limit $w \rightarrow \infty$, DURW is equivalent to $\mathrm{UNI}$ ).

\section{Results}

The first simulation results are presented in Figures 1$3 d$. Figure 1 shows three sample paths with estimates of $\phi_{1000}$ (y-axis) using DURW (using random jump weights $w \in\{0.1,1,10\})$ and UNI. The $\mathrm{x}$-axis shows the number of samples (in log scale). These results were obtained using the Youtube dataset with random sampling cost $c=1$. The choice of $\phi_{1000}$ is arbitrary; later we investigate estimation errors of all degrees. Note that none of the three runs of UNI can find a single node with outdegree 1000 until almost 200, 000 nodes have been sampled. On the other hand, DURW quickly finds at least one node with outdegree 1000, an useful property in scenarios where we are interested in finding high degree nodes. Moreover, DURW has consistently smaller estimation errors than UNI for most of the sample paths.

Figures 2-3d show estimates of the NMSE in log scale (over 1000 runs) of DURW for all outdegrees for different datasets. The NMSE error is obtained over 10,000 runs. In these simulations we compare the NMSE of DURW with the NMSE of the Metropolis-Hastings algorithm of Bar-Yossef et al. [3] and the NMSE of uniform random sampling (UNI). In all of the following simulations we sample $10 \%$ of the graph. The DURW random jump weight and cost are $w=10$ and $c=10$, respectively. Figures $2,3 \mathrm{a}, 3 \mathrm{~b}, 3 \mathrm{c}$, and $3 \mathrm{~d}$ show the NMSE for the Youtube, Wiki-Talk, Flickr, Livejournal, and Web-Google datasets, respectively.

We find that DURW obtains accurate estimates for all datasets (recall that an $\operatorname{NMSE}\left(\hat{\phi}_{j}\right) \leq 1$ characterizes an accurate estimate, according to our definition of "accurate", when $\phi_{j}$ is small, as the NMSE measures the relative error of $\hat{\phi}_{j}$ compared to the true value $\phi_{j}$ ). DURW outperforms all other methods w.r.t. the accuracy when estimating the fraction of nodes with large outdegrees, often by a factor of one order of magnitude. Moreover, DURW is significantly more accurate than Bar-Yossef et al. [3] over most outdegrees values. In the Youtube, Wiki-Talk, Flickr, and Livejournal datasets the value of $\hat{\phi}_{j}$ obtained with DURW for large outdegrees is up to two orders of magnitude more accurate than Bar-Yossef et al. [3]. DURW is also consistently more accurate over most outdegrees than UNI for the Youtube, Wiki-Talk and Flickr datasets and more accurate than UNI in the Livejournal and 
Web-Google datasets for large outdegrees (outdegrees larger than 50 in Livejournal and outdegrees larger than 30 in WebGoogle).

In Figures 2-3d we note that UNI is sometimes slightly better than DURW for small degrees, but not by much. This is because DURW is biased towards sampling nodes with large outdegrees and indegrees (these nodes tend to have large degrees in our constructed undirected graph). Thus, DURW tends to sample nodes with few outgoing edges less frequently than UNI, sometimes causing larger estimation errors for these small outdegree nodes. As we see later, increasing the DURW jump weight parameter $w$ reduces the estimation error for small outdegrees at the cost of increasing the error of larger outdegrees.

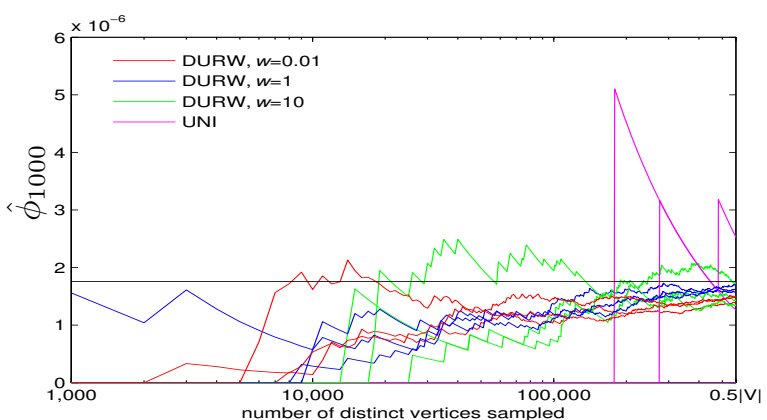

Figure 1: (YouTube) True value of $\phi_{1000}=1.8 \times 10^{-6}$ shown in the black solid line. Y-axis: Estimated $\phi_{1000}$ (DURW with $w \in 0.1,1,10$ and UNI). X-axis: Number of samples in $\log$ scale. DURW is consistently better than UNI.

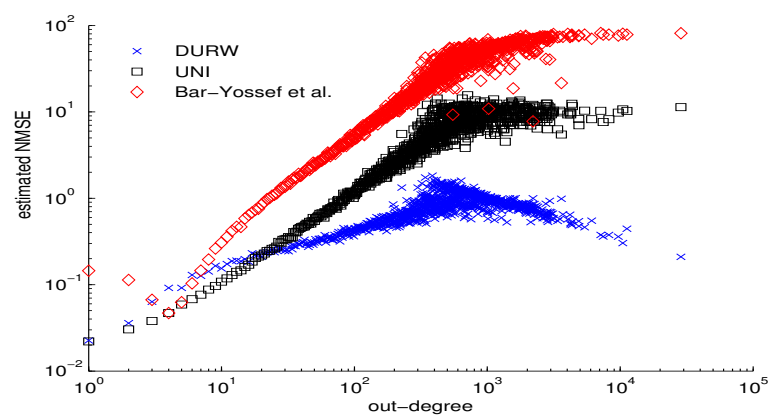

Figure 2: (YouTube) NMSE of DURW with $B 0.1|V|, w=10$, and $c=10$ compared with UNI.

\section{A. Varying DURW Parameters}

In what follows we study the impact of the DURW parameters on the accuracy of the estimates. Figure 4 shows the results of varying the random jump weight, $w$, while keeping the random jump cost $c=10$ and the sampling budget $B=0.1|V|$ fixed. The DURW parameter $w$ controls the tradeoff between the error for estimating small and large outdegrees. As $w$ increases, estimation errors for large outdegrees increase while errors for small outdegrees decrease. Conversely, as $w$ decreases the opposite happens, estimation errors for large outdegrees decrease while errors for small degrees increase. This is expected, as when $w$ increases DURW performs random jumps often and, thus, mimics uniform sampling (UNI), sampling small outdegrees nodes more often.

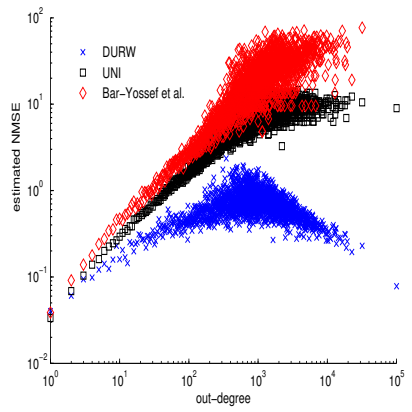

(a) Wiki-Talk

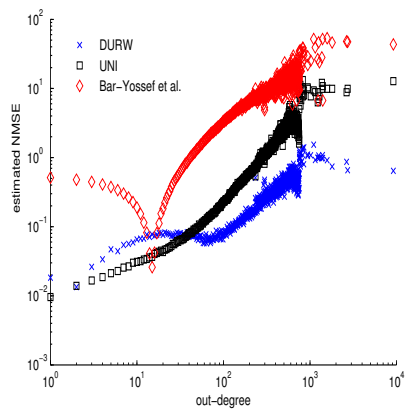

(c) LiveJournal

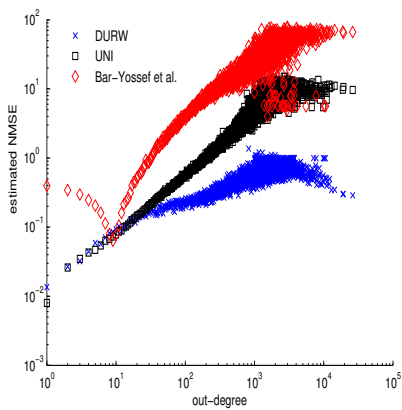

(b) Flickr

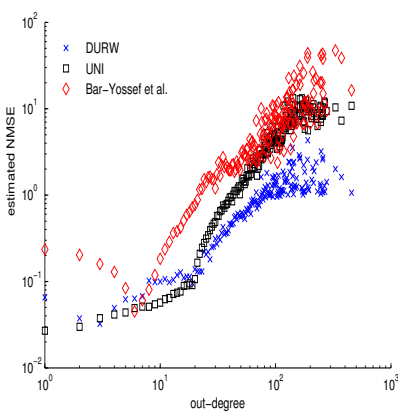

(d) Web-Google
Figure 3: NMSE of DURW with $B=0.1|V|, w=10$, and $c=10$ compared with UNI.

In the next set of simulations we look at the impact of the sampling budget, $B$, on the NMSE. Figure 5 presents the NMSE of Youtube for sampling budgets $B \in$ $\{0.01|V|, 0.1|V|, 0.2|V|\}$ with $c=10$ and $w=10$. We observe that the error of sampling $B$ nodes is roughly proportional to $1 / \sqrt{B}$. For instance, in Figure 5 we see that a one order of magnitude increase in $B$ roughly decreases the error by $1 / \sqrt{10}$.

As DURW performs random jumps, we also study the impact of the cost of these jumps on estimation accuracy. The cost of a jump is measured by the amount of "sampling budget" (queries) required to perform the jump. On some social networks, such as MySpace and Flickr, a number of queries is needed to sample a node uniformly at random. For instance, on Flickr random jumps are performed by querying randomly generating user IDs. The average number of random IDs queried until one valid ID is obtained is 77 to 1 (see our technical report [15]). The cost of a jump effectively reduces the number of total nodes that can be sampled, and, thus, increases the NMSE. Figure 6a shows the NMSE of Youtube with $c \in\{1,10,77\}$ and constant values $B=0.1|V|$ and $w=10$. Unsurprisingly, we observe that the estimation error of the outdegree distribution tail increases with $c$. This happens because decreasing $w$ also decreases the frequency of jumps, which then reduces the impact of parameter $c$ on the NMSE. To better understand this relationship, we repeat the above experiment (Figure 6a) with less frequent jumps $w=1$. In Figure $6 \mathrm{~b}$ we plot the NMSE of DURW with different values of $c \in\{1,10,77\}$ with $w=1$ constant. Comparing 
Figures $6 \mathrm{a}$ and $6 \mathrm{~b}$ we see that a smaller $w$ significantly lessens the negative impact of higher jump costs on the accuracy of the estimator.

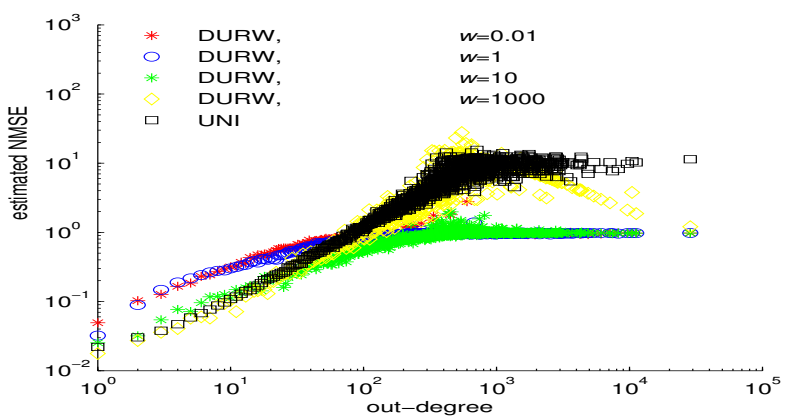

Figure 4: (Youtube) NMSE of DURW with $w \in$ $\{0.01,1,10,1000\}$ against the NMSE of UNI, $c=10$ and $B=0.1|V|$.

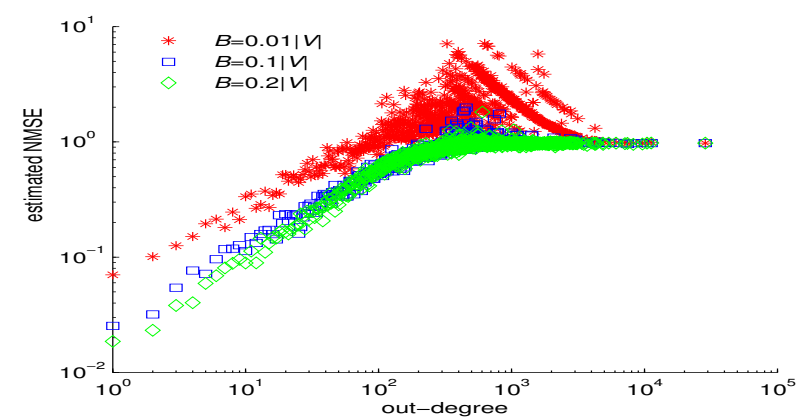

Figure 5: (Youtube) NMSE of DURW with $B \in$ $\{0.01|V|, 0.1|V|, 0.2|V|\}, c=10$ and $w=10$.

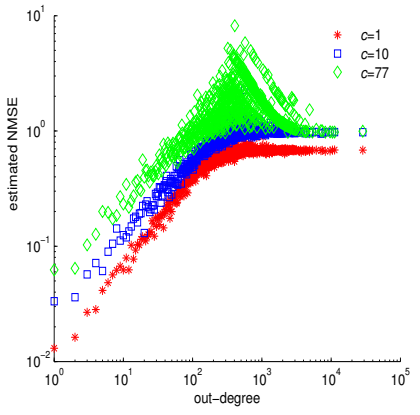

(a) $w=10$

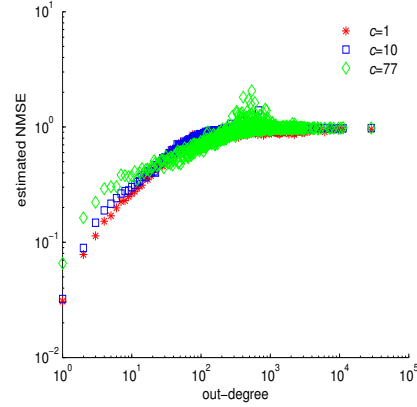

(b) $w=1$
Figure 6: (Youtube) NMSE of DURW with $c \in\{1,10,77\}$, $B=0.1|V|$ and $w=\{1,10\}$.

\section{Applications}

In this section we describe some experiments performed to estimate other metrics evaluated at the Wikipedia network. As opposed to the results presented in the previous section, which are based on datasets, we now use a DURW to crawl the graph in an online fashion.

These metrics are interesting if measured online: (1) the time between two consecutive article (node) revisions and (2) the distribution of the number of revisions in the last 7 days, classified by the outdegree of the node (article). But why do we want to sample Wikipedia revisions using DURW? Articles that point to several other articles are likely to suffer more revisions than nodes with almost no references. As DURW can accurately estimate metrics of nodes with high outdegrees (see Section IV), DURW is well equipped to estimate revisionrelated metrics.

Wikipedia provides a query API that can be used to obtain information from an node such as the categories to which it belongs, its revision timestamps (timestamps marking when the node was changed) and the content itself, which includes the text describing the node and links to other Wikipedia nodes. We implemented a crawler that uses this query API to perform a DURW random walk on the Wikipedia graph. For the DURW uniform random jumps we use the handy "show me a random article" function of the Wikipedia API. We set the DURW jump weight to $w=0.1$, allowing the walker to perform some random jumps while preserving most of the random walk characteristic of sampling high degree nodes. We are interested in sampling high degree nodes well because these nodes, although rare, are likely to be the ones that are edited more frequently. Our DURW crawler collected 79,708 Wikipedia nodes (approximately $2 \%$ of the entire Wikipedia database) over the course of two days (from 07/27/2011 5pm until 07/29/2011 5pm).

When an node is visited, the crawler also gathers measures of interest such as the outdegree and the times of the 500 most recent revisions. For conciseness, we omit the results regarding the outdegree (see Section III-F for a description of how to estimate the outdegree distribution). In particular, the metrics we obtain include:

- $X(v)$ : number of revisions of $v$ created in date $d \in[R(v)-$ 7 days, $R(v)]$, and

- $Y(v)$ : time (in days) that has passed between the last revision of $v$ and $R(v)$,

where $R(v)$ is the time when the node $v$ was first retrieved.

Following the estimator presented in Section III-F, we adapt equation (3) to estimate the distribution of the number of revisions in the last 7 days (denoted recent revision) since the node was first accessed binned by node outdegree. We group the node outdegrees in 6 bins such that the fraction of nodes (in the entire Wikipedia) is roughly the same in each bin. Note that we use the estimated outdegree distribution to specify the bins. Hence, we have

$$
h_{(b, j)}(v)= \begin{cases}1 & \text { node } v \text { in bin } b \text { has } j \text { recent revisions, } \\ 0 & \text { otherwise. }\end{cases}
$$

Figure 7 plots the average value of $R$ in each of the 6 bins. It also shows a "zoom-in" of the last bin by splitting it into 5 bins, without keeping a similar fraction of nodes per bar (it now varies from $0.006 \%$ to $11 \%$ of the entire Wikipedia). Observe that the nodes with the highest outdegrees are also those which are update more often. High outdegree nodes are clearly important in Wikipedia.

Figure 8 depicts the estimated CCDF of $Y$ as a solid red line. Note that the majority of the nodes were updated in the last 100 days. Now suppose that we want to obtain an estimate of the time between revisions at the instant we are sampling 


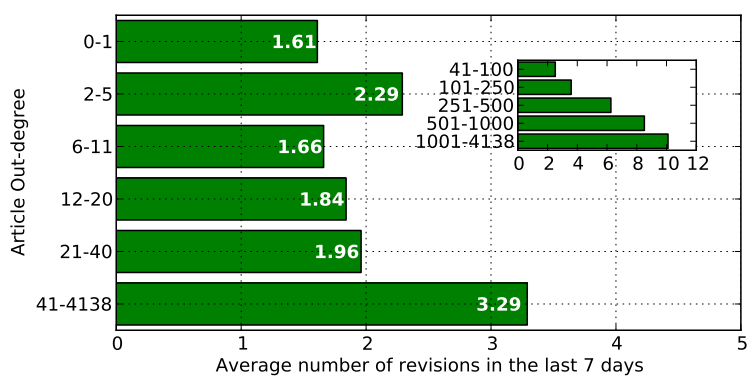

Figure 7: (Wikipedia) Average number of revisions in the last 7 days binned by node outdegree.

Wikipedia. The difference between the average time between revisions in a long period of time and the instantaneous time between revisions is parallel to the difference between average speed of a car over, say, 100 miles and its instantaneous velocity.

Note that $Y$ is not the time between revisions due to the inspection paradox, described as follows. Let $Z$ be the time between revisions (in days) at the time we sample Wikipedia. Let $\zeta_{z}=P[Z=z]$ and $\zeta_{\geq z}=P[Z \geq z]$. Assume that we arrive to sample an node uniformly between two revisions (a reasonable assumption). The probability of landing on an inter-revision time of size $Z=z$ is $\left(z \zeta_{z}\right) /\left(\sum_{k=1}^{\infty} k \zeta_{z}\right)$. Now we can relate $Y$ (the residual time) to $Z$

$$
P[Y=y]=\sum_{z=y}^{\infty} \frac{1}{z} \frac{\zeta_{\geq z}}{E[Z]}
$$

The maximum log-likelihood estimator of $\zeta_{z}$ is

$$
\underset{\left\{\hat{\zeta}_{z}\right\}_{\forall z}}{\operatorname{argmax}} \sum_{\forall y} f(y) \frac{1-\sum_{z=1}^{y-1} \hat{\zeta}_{z}}{\sum_{k=1}^{\infty} k \hat{\zeta}_{k}},
$$

where $f(y)$ is the number of samples of $Y$ with value $y$. We also need to enforce the constraints $0 \leq \hat{\zeta}_{z} \leq 1, z=1,2, \ldots$. and $\sum_{\forall z} \hat{\zeta}_{z}=1$. Figure 8 shows the estimated distribution $\hat{\zeta}_{z}$. Observe that more than $10 \%$ of the nodes have time between revisions greater than 100 days.

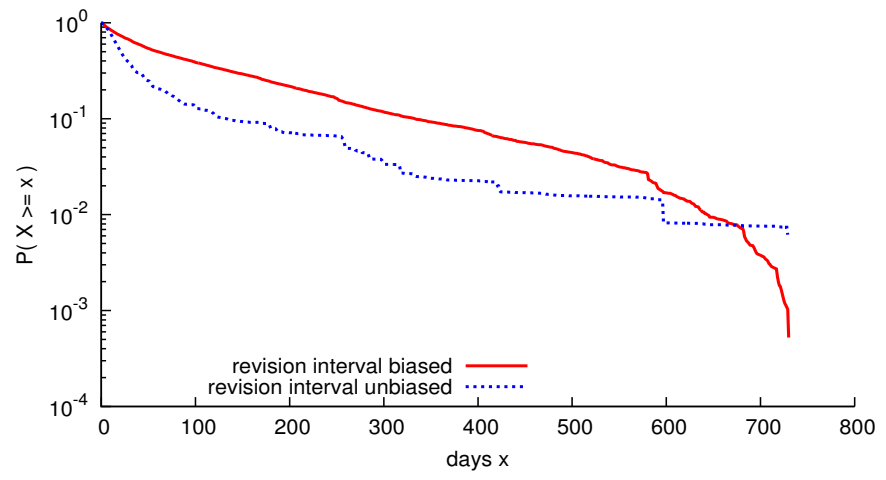

Figure 8: (Wikipedia) Estimated distribution of time between revisions. The red solid curve shows the raw results obtained by the DURW algorithm and the green dashed curve shows the results with their inspection paradox bias removed.

\section{Estimating Latent In-DEgRee Distributions}

The approach used above to estimate the outdegree distribution can also be used to estimate the indegree distribution if indegrees are visible to the random walker. In the presence of latent indegrees, , i.e., when an incoming edge $v \rightarrow u$ to node $u \in V$ is only observed from node $v$, estimating the indegree distribution is much more challenging. In such scenario the indegree distribution is a latent graph characteristic. A latent graph characteristic is one that cannot be directly observed but is rather inferred (through a mathematical model) from other observable variables.

In this section we show how to estimate the indegree (outdegree) distribution when indegrees (outdegrees) are latent. Unfortunately, our results are mostly negative. We observe that one needs to sample a large fraction of the incoming edges in the graph in order to obtain accurate estimates. The walker observes an incident edge $v \rightarrow u$ by sampling node $v \in V$.

A model that fully captures how a random walk samples edges on a directed graph does not yeild a mathematicaly tractable estimator as all sampled edges are correlated. Thus, in what follows we make the following simplifying assumption: Incoming edges sampled by a random walker are sampled independently and with probability $p$, where $p$ is a constant. This is a reasonable assumption as in undirected graphs, a walker tends to explore new nodes in the beginning instead of revisiting old nodes [12]. Moreover, although edges sampled by a random walk are not independent in our experience independent edge sampling provides a good model to study random walk properties.

Sampling incoming edges allows us to estimate the original indegree distribution as follows. Let $W$ be the maximum indegree of $G_{d}$. Let $Y$ denote the number of incoming edges to a node and

$$
c_{j i} \equiv P(X=j \mid X>0, Y=i)=\frac{\left(\begin{array}{l}
i \\
j
\end{array}\right) p^{j} q^{i-j}}{1-q^{i}}
$$

be the conditional probability of sampling $j(j=1, \ldots W)$ incoming edges in a node with indegree $i(i \geq j)$ given that at least one edge is sampled (i.e., $j \geq 1$ ). Denote $d_{j}$ the probability that a observed node has exactly $j$ sampled edges

$$
d_{j}=P(X=j \mid X>0)=\sum_{i=j}^{W} c_{j i} \theta_{i} .
$$

Or, in matrix notation,

$$
d=C \theta,
$$

where $d=\left(d_{1}, \ldots, d_{W}\right)^{\top}$ and $C=\left[c_{j i}\right], j, i=1, \ldots, W$. Here $c_{j i}=0$ whenever $j>i$. A way to estimate $\theta$ from $d$ uses the likelihood function

$$
f(j, \theta)=d_{j} .
$$

By maximizing equation (6) using the derivative of the log likelihood, $\partial \ln f(j \mid \theta) / \partial \theta_{i}=b_{j i} / d_{i}$, we have a Maximum Likelihood Estimator (MLE) of $\theta$.

To test the accuracy of our MLE we perform a set of experiments. Let $W=50$ (i.e., we remove vertices with 
more than 50 incoming edges from the graph). The value of $W$ is chosen arbitrarily. Figure 9 shows the true indegree distribution of the Flickr network (black line with asterisk) and the indegree distribution estimates for different sampling probabilities $p \in\{0.1,0.5,0.9\}$. Note that while the estimates based on a number of samples covering $90 \%$ of the edges $(p=0.9)$ are reasonable for small indegrees (but still not accurate for higher degrees), the estimates with sampling probabilities $p=0.1$ and $p=0.5$ are quite inaccurate.

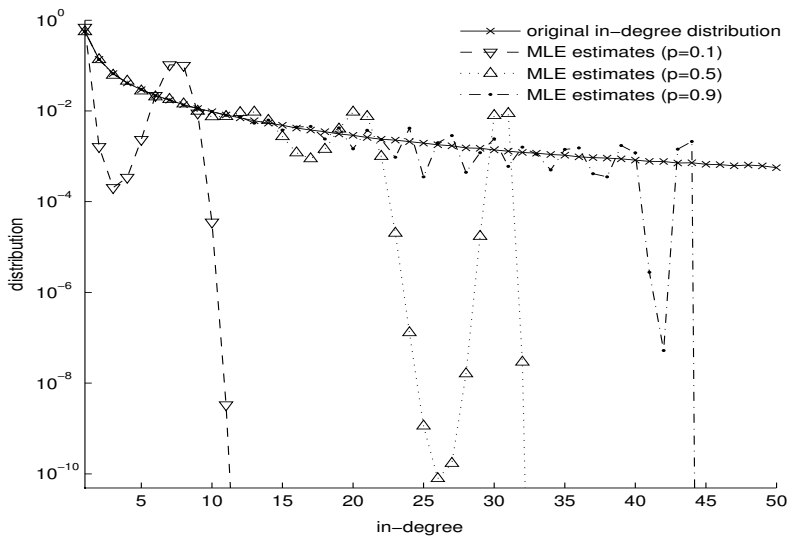

Figure 9: (Flickr) MLE indegree distribution estimates for $p=$ $0.1,0.5$, and 0.9 .

The experiment in Figure 9 indicate that indegree distribution estimates are inaccurate if $p$ is small. In our technical report [15] we complement the above empirical observation with a theoretical lower bound of the mean squared error of any unbiased indegree distribution estimator. Our theoretical results agree with the experimental results shown in Figure 9. Adding additional side information, for instance, that the average indegree equals the average outdegree, yields little improvement.

Many directed graphs are characterized by symmetric edges. For instance, in the Web-Google dataset $69 \%$ of the edges are symmetric (the symmetry of other datasets is summarized in Table I). In a graph where all edges are symmetric (i.e., every edge $(u, v) \in E_{d}$ has a corresponding edge $(v, u) \in$ $E_{d}$ ) the indegree and the outdegree distributions are the same. In what follows we consider a model that takes advantage of symmetry to improve the indegree distribution estimates. Unfortunately, our experiments show that while moderate edge symmetry increases estimation accuracy, it is still insufficient to obtain accurate estimates.

\section{A. Side Information: Edge Symmetry}

Consider a directed graph $G_{d}=\left(V, E_{d}\right)$. Let $s$ denote the fraction of symmetric edges in $E_{d}$, where $s=1$ when all edges in $G_{d}$ are symmetric. Edge symmetry can convey information about the indegree distribution. For instance, if $s=1$ the indegree distribution is equivalent to the outdegree distribution. To assess the increase in estimation accuracy that comes from the presence of symmetric edges, consider the following model.

Let $v$ be a sampled vertex. Consider the following random variables associated with $v$ :
- $Z$ : indegree.

- $Z_{s}$ : number of symmetric incoming edges.

- $Z_{a}$ : number of incoming asymmetric edges.

- $Y$ : observed outdegree.

- $X_{s}$ observed number of symmetric incoming edges.

- $X_{a}$ observed number of asymmetric incoming edges.

Let $\rho(y, z)=P[Y=y, Z=z]$ denote the joint indegree and outdegree distribution of $v, p$ the sampling rate, and $\alpha$ the fraction of symmetric edges. We assume that the number of outgoing edges of $v$ that are symmetric is a Binomial random variable with parameter $\alpha$,

$$
\begin{aligned}
& P\left[Z_{s}=z_{s} \mid Y=y, Z=z\right]= \\
& \left\{\begin{array}{cl}
\left(\begin{array}{c}
\min (y, z) \\
z_{s}
\end{array}\right) \alpha^{z_{s}}(1-\alpha)^{\min (y, z)-z_{s}} & \text { if } z_{s} \leq \min (y, z), \\
0 & \text { otherwise. }
\end{array}\right.
\end{aligned}
$$

We seek to find a likelihood function of the observed random variables $Y, X_{s}$, and $X_{a}$ with respect to $\rho, P\left[Y=y, X_{s}=\right.$ $\left.x_{s}, X_{a}=x_{a} \mid \rho\right]$. Note that

$$
\begin{aligned}
P & {\left[Y=y, X_{s}=x_{s}, X_{a}=x_{a} \mid \rho\right] } \\
=\sum_{\forall z} P\left[X_{s}=x_{s}, X_{a}=x_{a} \mid Y=y, Z=z\right] \rho_{y, z} & \\
=\sum_{\forall z} \rho_{y, z} \sum_{z_{s}=0}^{z} P\left[X_{s}=x_{s}, X_{a}\right. & \left.=x_{a} \mid Z_{s}=z_{s}, Y=y, Z=z\right] \\
& \times P\left[Z_{s}=z_{s} \mid Y=y, Z=z\right],
\end{aligned}
$$

where

$$
\begin{aligned}
& P\left[X_{s}=x_{s}, X_{a}=x_{a} \mid Z_{s}=z_{s}, Y=y, Z=z\right] \\
& =P\left[X_{s}=x_{s}, X_{a}=x_{a} \mid Z_{s}=z_{s}, Y=y, Z_{a}=z-z_{s}\right] \\
& =\left(\begin{array}{c}
z_{s} \\
x_{s}
\end{array}\right) p^{x_{s}}(1-p)^{z_{s}-x_{s}}\left(\begin{array}{c}
z-z_{s} \\
x_{a}
\end{array}\right) p^{x_{a}}(1-p)^{z-z_{s}-x_{a}} \\
& =\left(\begin{array}{c}
z_{s} \\
x_{s}
\end{array}\right)\left(\begin{array}{c}
z-z_{s} \\
x_{a}
\end{array}\right) p^{x_{s}+x_{a}}(1-p)^{z-x_{s}-x_{a}}
\end{aligned}
$$

with $P\left[Z_{s}=z_{s} \mid Y=y, Z=z\right]$ as defined in equation (7).

In what follows we introduce the Fisher information matrix. The inverse of the Fisher information matrix gives a lower bound on the mean squared error of any unbiased estimate of the indegree distribution. This lower bound is known as the Cramér-Rao bound. The indegree distribution Fisher information associated with the symmetric edge information can be computed from the Fisher information of $P[Y=$ $\left.y, X_{s}=x_{s}, X_{a}=x_{a} \mid \rho\right]$ with respect to $\rho$ by noting that $\theta$, the indegree distribution, can be defined as $\theta_{z}=\sum_{\forall y} \rho(y, z), \forall z$, or in matrix form $\theta=H \rho^{\top}$, where $\rho=(\rho(1,1), \rho(2,1), \ldots)$ and

$$
H=\left[\begin{array}{lllllll}
1 & \ldots & 1 & & & & \\
& & & \ldots & & & \\
& & & & 1 & \ldots & 1
\end{array}\right] .
$$

Let $J_{\rho}$ denote the Fisher information with respect to the joint distribution $\rho$. Computing $J_{\rho}$ from $P\left[Y=y, X_{s}=x_{s}, X_{a}=\right.$ $\left.x_{a} \mid \rho\right]$ is trivial. Let $J_{\theta}$ denote the Fisher information with 


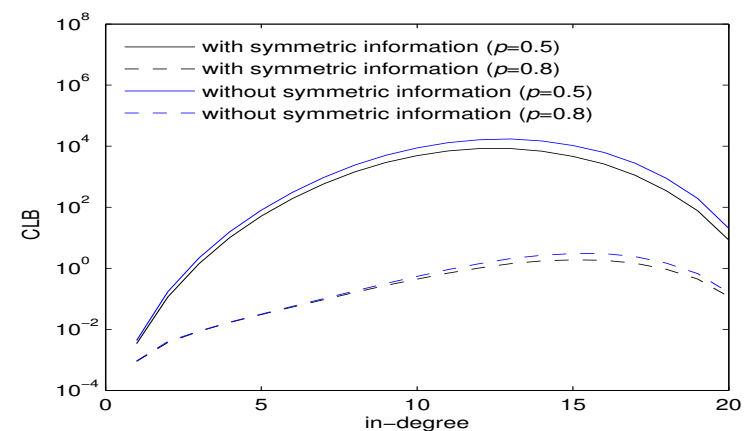

Figure 10: (Flickr) Square root of the Cramér-Rao lower bound normalized by the true value of $\theta$. The curves show the error lower bound with and without symmetric edge information. Edge symmetry at $\alpha=62 \%$. Lower is better.

respect to the indegree distribution $\theta$. Then [17, pages 83-84]

$$
J_{\theta}=H J_{\rho} H^{\top} .
$$

Matrix $J_{\theta}$ encodes the information obtained from the observed incoming edges plus the information that the graph is symmetric. We do this inversion numerically in Section VI-B and observe that adding symmetric information does not significantly improve the estimation error unless most edges in the graph are symmetric.

\section{B. Numerical Results}

In the following experiment we include symmetry information in the Cramér-Rao lower bound computed by inverting $J_{\theta}$ (which is a bound on the mean squared error of any unbiased estimator of $\theta$ ). Figure 10 shows the square root of the CramérRao lower bound divided by the true value of $\theta$ (CLB) of Flickr for maximum indegree $W=20$, with and without Flickr's symmetric information. In Flickr the fraction of edges that are symmetric is $\alpha=0.62$. Observe that while symmetry reduces the Cramér-Rao lower bound, it is not enough to significantly increases the estimation accuracy to acceptable levels. Moreover, other experiments (not shown here) indicate that increasing $W$ significantly increases the estimation error (to the point that even estimating $\theta_{1}$ can be made inaccurate).

\section{RELATED WORK}

Estimating observable characteristics by sampling a directed graph (in this case, the Web graph) has been the subject of Bar-Yossef et al. [3] and Henzinger et al. [8], which transform the directed graph of web-links into an undirected graph by adding reverse links, and then use a Metropolis-Hastings RW to sample webpages uniformly. Our "backward edge traversal" is an adaptation of the method of Bar-Yossef et al. [3] to work with a pure random walk and random jumps. Both of these Metropolis-Hastings RWs are designed to sample directed graph that do not allow random jumps. However, in the presence of random jumps (even if jumps are rare), the MetropolisHastings RW algorithm is not as efficient and as accurate as our DURW algorithm. Random walks with PageRank-style jumps are used in Leskovec and Faloutsos [9] to sample large graphs. In Leskovec and Faloutsos [9], however, there is no technique to remove the large biases induced by the random walk and the random jumps, which makes this method unfit to estimate graph characteristics. In contrast, our distribution estimates are asymptotically unbiased.

\section{CONCLUSiOnS \& Future WorK}

In this work we provide the first random walk method to accurately estimate characteristics of directed graphs that allow random jumps. Also, to the best of our knowledge our work is the first to study and provide a sound theoretical analysis of the problem of estimating latent indegree distributions. Our DURW has a single parameter, $w$, which controls how often the random walk needs to perform an uniform random jump. From our experience we observe that a value of $w$ that is no less than half and no more than twice the average outdegree yields good results. Our future work includes reducing the transient and exploring the parameter space of the DURW algorithm.

\section{ACKNOWLEDGMENT}

This research was sponsored by the NSF under CNS-1065133, ARO under MURI W911NF-08-1-0233, and the U.S. Army Research Laboratory under Cooperative Agreement W911NF-09-2-0053. The views and conclusions contained in this document are those of the authors and should not be interpreted as representing the official policies, either expressed or implied of the NSF, ARO, ARL, or the U.S. Government. The U.S. Government is authorized to reproduce and distribute reprints for Government purposes notwithstanding any copyright notation hereon.

\section{REFERENCES}

[1] Google Programming Contest, 2002.

[2] Predicting Positive and Negative Links in Online Social Networks, 2010.

[3] Ziv Bar-Yossef and Maxim Gurevich. Random sampling from a search engine's index. J. ACM, 55(5):1-74, 2008.

[4] S. Brin and L. Page. The anatomy of a large-scale hypertextual Web search engine. In Proc. of the WWW, 1998.

[5] Facebook. http://www.facebook.com, 2010.

[6] Flickr. http://www.flickr.com, July 2010.

[7] Douglas D. Heckathorn. Respondent-driven sampling: A new approach to the study of hidden populations. Social Problems, 1997.

[8] Monika R. Henzinger, Allan Heydon, Michael Mitzenmacher, and Marc Najork. On near-uniform url sampling. In Proceedings of the $W W W$, pages 295-308, 2000.

[9] Jure Leskovec and Christos Faloutsos. Sampling from large graphs. In Proc. of the KDD, pages 631-636, 2006.

[10] Alan Mislove, Massimiliano Marcon, Krishna P. Gummadi, Peter Druschel, and Bobby Bhattacharjee. Measurement and Analysis of Online Social Networks. In Proc. of the IMC, October 2007.

[11] Amir H. Rasti, Mojtaba Torkjazi, Reza Rejaie, Nick Duffield, Walter Willinger, and Daniel Stutzbach. Respondent-driven sampling for characterizing unstructured overlays. In Proc. of the IEEE Infocom, pages 2701-2705, April 2009.

[12] Bruno Ribeiro, Prithwish Basu, and Don Towsley. Multiple random walks to uncover short paths in power law networks. Technical Report UM-CS-2011-049, UMass Amherst, 2011.

[13] Bruno Ribeiro, William Gauvin, Benyuan Liu, and Don Towsley. On MySpace account spans and double Pareto-like distribution of friends. In Proceedings of the IEEE Infocom NetSciCom Workshop, 2010.

[14] Bruno Ribeiro and Don Towsley. Estimating and sampling graphs with multidimensional random walks. In Proc. of the IMC, 2010.

[15] Bruno Ribeiro, Pinghui Wang, Fabricio Murai, and Don Towsley. Sampling directed graphs with random walks. Technical Report UMCS-2011-031, UMass Amherst, 2011.

[16] D. Stutzbach, R. Rejaie, N. Duffield, S. Sen, and W. Willinger. On unbiased sampling for unstructured peer-to-peer networks. IEEE/ACM Trans. Netw., 17(2):377-390, 2009.

[17] Hary L. van Trees. Estimation and Modulation Theory, Part 1. Wiley, New York, 2001.

[18] Wikipedia website. http://www.wikipedia.org, 2010. 